\title{
Multivisceral resection for growing teratoma syndrome: overcoming pessimism
}

\author{
Mukur Dipi Ray, MS; ${ }^{a}$ Bharat Bhushan Khurse, MS; ${ }^{a}$ Tapan Chauhan, MS; ${ }^{a}$ Ashish \\ Jakhetiya, $\mathrm{MCh} ;{ }^{\mathrm{a}}$ and Pankaj Kumar Garg, $\mathrm{MCh}^{\mathrm{ab}}$
}

${ }^{a}$ Department of Surgical Oncology, Dr BR Ambedkar Institute Rotary Cancer Hospital, All India Institute of Medical Sciences, New Delhi, India; and ${ }^{b}$ Department of Surgery, University College of Medical Sciences and Guru Teg Bahadur Hospital, University of Delhi, India

G rowing teratoma syndrome (GTS) is a rare condition seen in patients with germ-cell tumors (GCT) who present with enlarging masses during or after appropriate chemotherapy with normalized serum markers. ${ }^{1}$ Three defining criteria for GTS are: a persistently growing tumor mass or evolving new mass during or after chemotherapy, normalization of tumor markers, and presence of only mature teratoma in the resected specimen on the final histopathological examination. ${ }^{1}$ Growing teratomas lack the metastatic potential; however, their relentless local growth causes compression and infiltration of adjacent organs, which produces symptoms. These tumors may attain huge size with passage of time if they are left untreated. Surgery is the only potentially curative option for GTS as these growing teratomas are chemotherapy and radiotherapy resistant. Here we report a case of GTS in a 30-year-old woman for 2 reasons: first, to report one of the largest-known growing teratomas - it weighed $17.2 \mathrm{~kg}(38 \mathrm{lb})$ after resection; and second, to emphasize that complete surgical excision should be the aim of surgery, which may include multivisceral resection. No patient should be deemed unresectable unless surgical exploration has been undertaken at a high-volume center, which has expert surgical and anesthetic facilities.

\section{Case presentation and summary}

A 30-year-old woman presented with an abdominal lump that had been increasing progressively during the previous 2 months. She had been well until 5 months before presentation to us when she developed lower abdominal pain. At that presentation, a pelvic ultrasonography revealed a right adnexal mass. She underwent right salpingo-oopherectomy in a community hospital. Histopathological exami- nation revealed mixed GCT. Her serum alpha-fetoprotein (AFP) and beta human chorio-gonadotrophic hormone $(\beta-\mathrm{HCG})$ levels were normal, but her lactate dehydrogenase (LDH) level was mildly elevated (400 U/L; normal, 105-330 U/L). She received 4 cycles of systemic chemotherapy (bleomycin $10 \mathrm{IU} / \mathrm{m}^{2}$ days $1-3$, etoposide $100 \mathrm{mg} / \mathrm{m}^{2}$ days $1-5$, and cisplatin $20 \mathrm{mg} / \mathrm{m}^{2}$ days $1-5$ ) and she remained asymptomatic for 2 months but to develop the progressively increasing abdominal lump again.

A contrast-enhanced computed-tomography scan of the abdomen showed large multiple heterogenous intraperitoneal masses; there was infiltration of bladder and bowel (Figure 1). Sigmoidoscopy and cystoscopy confirmed bladder and colon infiltration. Her tumor markers were normal. Image-guided biopsy was consistent with teratoma only - no immature component was found. She was deemed unresectable at many other oncology centres and was referred to our centre for best supportive and palliative care as a case of unresectable growing teratoma syndrome.

At our center, we advised surgery because it is the only potentially curative option. Surgical exploration revealed multiple intraperitoneal masses, the largest of which was $30 \times 25 \times 20 \mathrm{~cm}(12 \times 10 \times 9$ inches). A few of them were found to be infiltrating the sigmoid colon and urinary bladder. Complete $\mathrm{R}^{\prime} 0$ ' resection could be achieved - excision of all masses with resection of the involved colon and subtotal cystectomy was undertaken (Figure 2). Bowel continuity was achieved through colorectal anastomosis and urinary bladder augmentation was done through iliocystoplasty. Her postoperative period was uneventful. The total weight of the resected specimens was $17.2 \mathrm{~kg}$ (38 lb). Histopathological examination confirmed mature teratoma with no

Accepted for publication July 17, 2015. Correspondence: Pankaj Kumar Garg, MCh; dr.pankajgarg@gmail.com. Disclosures: The authors report no disclosures or conflict of interests. JCSO 2016;14:320-322. @2016 Frontline Medical Communications. doi: 10.12788/jcso.0185. 

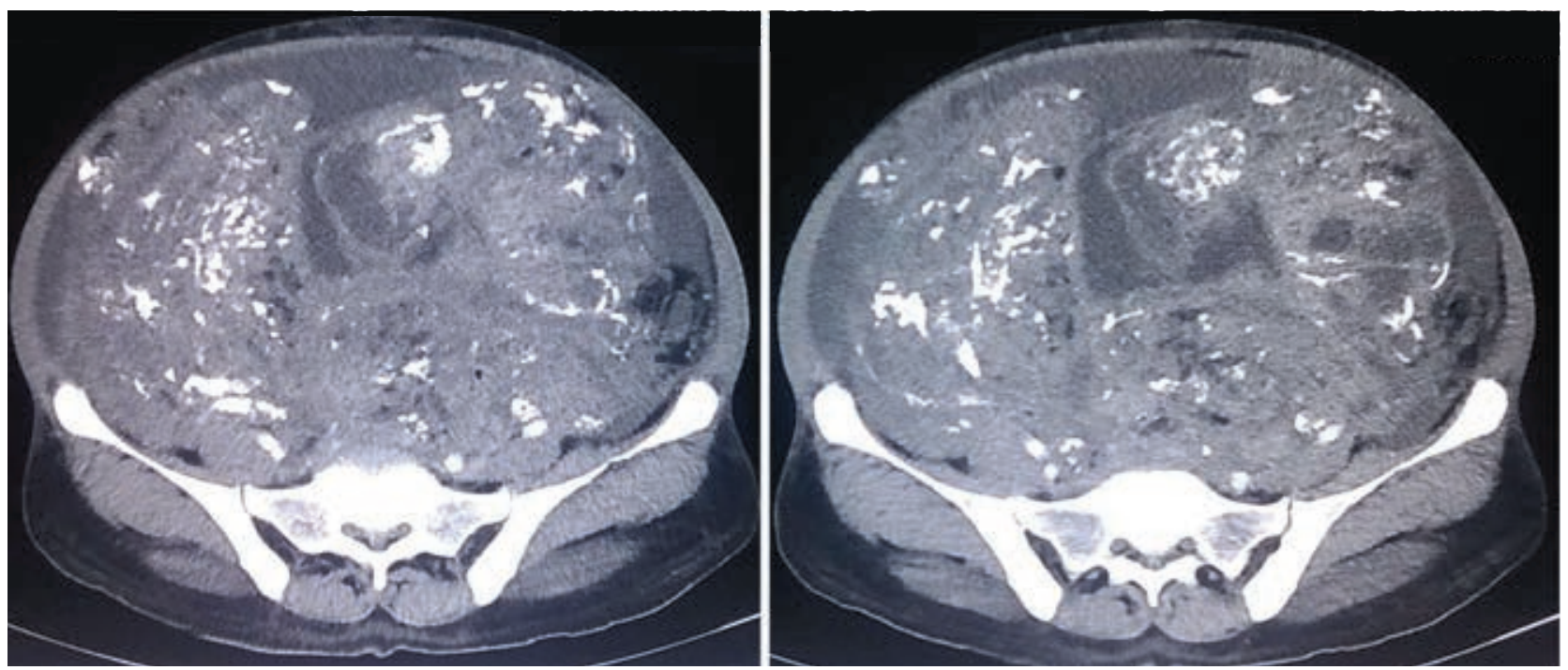

FIGURE 1 Axial sections of the contrast-enhanced computed-tomography scan of the abdomen and pelvis show a large intra-abdominal mass infiltrating the large bowel and urinary bladder.
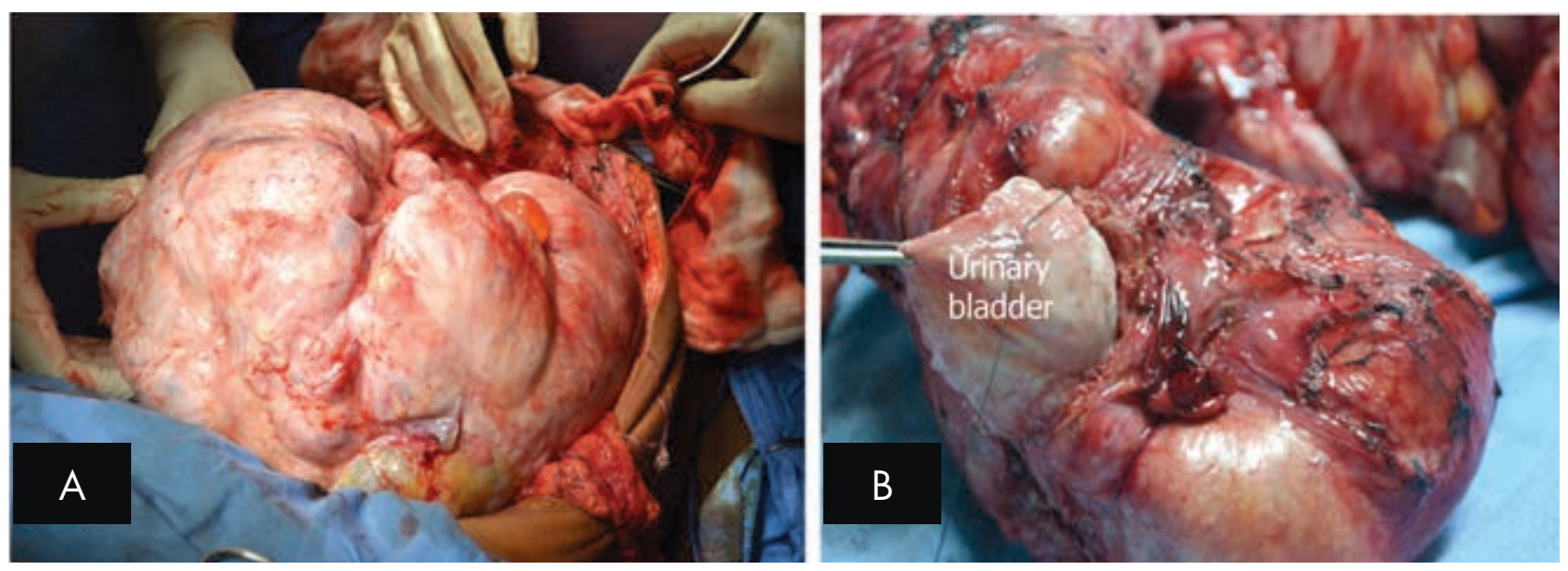

FIGURE 2 A, Intra-operative image showing large intra-abdominal mass. B, Postresection specimen.

immature component. She is under active surveillance and asymptomatic with no evidence of recurrence at 10 months of follow-up.

\section{Discussion}

Growing teratoma syndrome is a rare condition seen in patients with primary nonseminomatous germ-cell tumors (NSGCT) who present with enlarging masses during or after appropriate chemotherapy with normalized serum markers. ${ }^{1}$ The syndrome was first described by Logothetis and colleagues in $1982^{2}$ when they reported 6 cases of enlarging masses after receiving chemotherapy for testicular NSGCT with normalized tumor markers. All of the patients underwent surgical excision and were found to have mature teratoma with no viable malignant tumor on histopathological examination. It needs to be highlighted that a similar condition had already been described by DiSaia and colleagues in an ovarian GCT in 1977 well before Logothetis and colleagues described testicular GCT, but it had been "chemotherapeutic retroconversion." ${ }^{3}$ DiSaia and colleagues reported 3 cases of malignant ovarian GCT who were initially treated with VAC (vincristine, actinomycin D, and cyclophosphamide); histopathological examination of the resected specimen following second-look laparotomy revealed only mature teratomatous tissue. DiSaia and colleagues suggested 2 plausible explanations for this phenomenon of GTS: either malignant immature teratomatous tissue had converted into mature tissue because of chemotherapy, 
or chemotherapy could destroy only immature teratomatous tissue leading to growth of mature chemoresistant teratomatous tissue. Amsalem and colleagues described the case of a 12-year-old girl who developed GTS after completion of chemotherapy for malignant ovarian teratoma and suggested that GTS and chemotherapeutic retroconversion are perhaps different names of same phenomenon. ${ }^{4}$ Contrary to that view, Djordjevic and colleagues proposed that the terms are not synonymous. ${ }^{5}$ They argued further that mature teratomatous tissue in GTS has the ability to grow in size, unlike chemotherapeutic retroconversion, in which mature teratomatous nodules do not grow.

Relentlessly growing teratoma leads to symptoms caused by compression of adjacent organs. Huge size of the abdominal lump in our patient made her bedridden because of its sheer weight and compression of diaphragm. Though radiological features are not very reliable for its diagnosis, GTS can manifest as intraperitoneal masses with an increased fatty or cystic component in a patient with a history of a germ-cell tumor with normal tumor markers, during or after completion of chemotherapy. ${ }^{6,7}$

Surgery is the standard treatment for GTS as these

\section{References}

1. De Cuypere M, Martinez A, Kridelka F, Balague G, Maisongrosse V, Ferron G. Disseminated ovarian growing teratoma syndrome: a case report highlighting surgical safety issues. Facts Views Vis ObGyn. 2014;6:250-253.

2. Logothetis CJ, Samuels ML, Trindade A, Johnson DE. The growing teratoma syndrome. Cancer. 1982;50:1629-1635.

3. DiSaia PJ, Saltz A, Kagan AR, Morrow CP. Chemotherapeutic retroconversion of immature teratoma of the ovary. Obstet Gynecol. 1977;49:346-350.

4. Amsalem H, Nadjari M, Prus D, Hiller N, Benshushan A. Growing teratoma syndrome vs chemotherapeutic retroconversion: case report growing teratomas are notoriously resistant to chemotherapy and radiotherapy. Though these growing teratomas lack the potential for metastasis, they continue to grow relentlessly and involve adjacent organs if they are left untreated. Longstanding teratomas can also have malignant transformation. Complete surgical excision should be the aim of surgery as frequency of local recurrence after partial excision is $50 \%-83 \%$, compared with $0 \%-4 \%$ when complete resection is obtained. ${ }^{1}$ Multivisceral resection may be required in huge growing teratomas that have invaded the adjacent organs, as happened with our patient.

Considering the huge size of the abdominal lump which had infiltrated our patient's adjacent organs (at $17.2 \mathrm{~kg}$ postresection, possibly one of the largest growing teratoma reported), she was deemed unfit for curative surgery at many other centres and was referred to our centre for best end-of-life care. We wish to highlight that patients with giant growing teratoma should not be judged to have unresectable disease unless proper surgical exploration has failed to achieve R'0' resection. These patients must be appropriately referred to high-volume centres, which are equipped to deal with these case scenarios.

and review of the literature. Gynecol Oncol. 2004;92:357-360.

5. Djordjevic B, Euscher ED, Malpica A. Growing teratoma syndrome of the ovary: review of literature and first report of a carcinoid tumor arising in a growing teratoma of the ovary: Am J Surg Pathol. 2007;31:1913-1918.

6. Han NY, Sung DJ, Park BJ, et al. Imaging features of growing teratoma syndrome following a malignant ovarian germ cell tumor. J Comput Assist Tomogr. 2014;38:551-557.

7. Panda A, Kandasamy D, Sh C, Jana M. Growing teratoma syndrome of ovary: avoiding a misdiagnosis of tumour recurrence. J Clin Diagn Res. 2014;8:197-198. 